\title{
Point: Chemosensitivity Assays Have a Role in the Management of Recurrent Ovarian Cancer
}

\author{
Julian C. Schink, MD, and Larry J. Copeland, MD, ${ }^{\mathrm{b}}$ Chicago, Illinois, and Columbus, Ohio
}

\author{
Key Words \\ Chemosensitivity assays, in vitro chemosensitivity testing, recur- \\ rent ovarian cancer, personalized medicine
}

\begin{abstract}
In this era of personalized medicine, patients with recurrent ovarian cancer deserve better than the $25 \%$ response rate that is associated with drugs selected based on clinical information alone. In the past decade, marked laboratory improvements have enabled chemosensitivity assay testing to yield a 0.70 correlation with response, and to accurately predict progression-free and overall survival. Compelling retrospective data supporting the use of this technology cannot be ignored while waiting for a cooperative group to test whether chemosensitivity assay should be used to direct salvage therapy. (JNCCN 2011;9:115-120)
\end{abstract}

For the estimated 21,880 women in the United States diagnosed this year with ovarian cancer, most can expect their disease to respond completely to therapy, but most will eventually experience relapse and require additional chemotherapy. ${ }^{1}$ The process of choosing treatment for recurrent ovarian cancer from the list of NCCN acceptable therapies is particularly unsophisti-

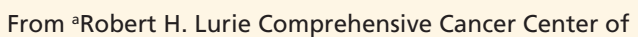
Northwestern University, Northwestern University Feinberg School of Medicine, Chicago, Illinois, and ${ }^{\mathrm{b}}$ The Ohio State University Comprehensive Cancer Center - James Cancer Hospital and Solove Research Institute, Columbus, Ohio.

Submitted September 16, 2010; accepted for publication November 2, 2010.

Dr. Schink has disclosed that he receives research support from Precision Therapeutics, Inc. Dr. Copeland has disclosed that he receives research support from Eli Lilly and Company; Genentech, Inc.; and Gynecologic Oncology Group. He is also on the advisory board or member of the speakers' bureau for Bayer HealthCare; Celgene Corporation; Eli Lilly and Company; Precision Therapeutics, Inc.; and sanofi-aventis U.S.

Correspondence: Julian C. Schink, MD, Robert H. Lurie

Comprehensive Cancer Center of Northwestern University,

Gynecologic Oncology Program, Northwestern Prentice Women's

Hospital, 250 East Superior Street, Suite 05-2168, Chicago, IL 60611.

E-mail: jschink@nmff.org cated, with platinum-free interval the most common selection criteria. As clinicians strive to ensure clinical benefit for patients, the time has come to partner with laboratory scientists to better predict which drugs are most effective and which will not provide benefit, thus potentially avoiding related toxicities.

Relying on laboratory testing to tailor therapy is the goal of personalized medicine. In his 2009 ASCO Presidential Address, Richard Schilsky commented,

As cancer specialists, we have long recognized that each patient is unique.... We now recognize that, just as each patient is different in how he or she is affected by cancer, each cancer has a distinctive biology and natural history, and every disease called "cancer" comprises smaller subsets with distinctive features and differing outcomes that require personalized treatment plans. ${ }^{2}$

An oncologist's obligation is to offer patients the best chance of success while avoiding toxicity associated with ineffective treatments. They should also make a concerted effort to provide the most cost-effective treatments available. This article reviews evidence supporting the use of chemosensitivity testing in the selection of treatment options for women with recurrent ovarian cancer to meet these obligations.

\section{History of Chemosensitivity Assay Testing}

In 1978, Salmon et $\mathrm{al}^{3}{ }^{3}$ described a soft agar culture system for bioassay of various tumor types. They presented early data on chemosensitivity assay testing in multiple myeloma and ovarian cancer cells. In the introduction they note, "Numerous clinical observations have shown a wide range of responsiveness to particular treatments among patients with cancers of identical histopathologic characteristics." Furthermore, they state, “...this assay would make predictive cancer chemotherapy fea- 
sible." This relatively small study had 9 cases of ovarian cancer, and all 3 women with sensitive tumors in vitro showing a partial response and all drug-resistant cases showing no response.

Subsequently, through the 1980s, different culture and testing techniques were evaluated and the potential value of these assays was better defined. The soft agar culture system and the clonogenic assay were found to have numerous technical problems, including poor reproducibility and the overgrowth of stromal and fibroblast cells, which were more likely interpreted as drug-resistant. In an editorial Von Hoff $^{4}$ stated,

It is difficult for anyone to determine why oncologists have not paid more attention to the use of in vitro predictive tests in the care of their patients. A review of already completed in vitroin vivo correlative trials in 2300 patients indicates percentages of 69 for true positives and 91 for true negatives from predictive assays. These percentages are as good as or better than those seen with already accepted tests, such as estrogen receptor assays or bacterial sensitivity testing systems.

Von Hoff then appealed to the clinical trials community to embrace and accrue patients to a prospective trial testing the merits of assay-directed therapy, but this did not happen.

Because these retrospective studies referenced by Von Hoff showed a greater than $90 \%$ negative predictive value, the concept of drug resistance testing was embraced. The Oncotech chemoresistance assay, known as the Extreme Drug Resistance (EDR) Assay, was rapidly adopted and became the market leader in the 1990s, promoting the concept that drug resistance could be more accurately predicted than drug sensitivity.

The success of the Oncotech EDR assay was both a blessing and a curse. It held the promise of personalized drug selection, with the goal of avoiding ineffective treatment. In 2003, a case-control study by Loizzi et al. ${ }^{5}$ showed a significant survival advantage for patients with ovarian cancer treated with assaydirected therapy. Unfortunately, this strategy of predicting failure is flawed, especially when applied to women with recurrent platinum-resistant ovarian cancer, because failure is already expected in $75 \%$ to 95\% of patients. The Oncotech EDR assay did not predict success, which was a major shortcoming be- cause personalized care must be about selecting drugs that provide clinical benefit.

In 2009, 2 large retrospective analyses critical of the Oncotech EDR assay were published. Karam et al. ${ }^{6}$ reviewed the clinical outcomes of 377 patients using multivariate analysis to identify independent prognostic factors with respect to time to progression, survival, and survival after recurrence. In this analysis, the commonly accepted prognostic factors such as optimal debulking and extent of residual disease were highly significant. However, EDR assay results for single-agent and combination regimens failed to predict any patient outcome. Matsuo et al. ${ }^{7}$ performed a retrospective analysis of the EDR Assay results for 253 women with advanced-stage ovarian cancer who had assay specimens submitted at their primary surgery. They found no statistical correlation between the number of drugs showing extreme resistance and chemotherapy response, progressionfree survival, or overall survival. On May 31, 2010, the California Medicare reimbursement panel disallowed coverage of the Oncotech EDR assay. In June 2010, Exiqon chose to terminate operations of their subsidiary, Oncotech, and marketing of the EDR assay was discontinued.

\section{Emergence of Chemosensitivity Testing}

In the continuing pursuit of personalized medicine, an individual patient's clinical benefit, rather than failure, must be the goal. Three new laboratory approaches to chemosensitivity testing emerged as the most promising in the past decade: the microplate ATP luminescence assay, the microculture (MiCK) assay for apoptosis, and the ChemoFx in vitro cell culture assay.

These proprietary assay systems all rely on the principles of industrial engineering to create highquality reproducible results, eliminating the technical limitations of prior assays, such as poor reproducibility, low evaluability rates, and excessive growth of stromal cells. The important steps that are generally common to these assays include careful disaggregation of tumor cells, selective culture to enrich the tumor population while minimizing the presence of stromal cells, characterization of the tumor cells, testing of cancer cell response to a wide range of therapeutic concentrations of the active form of the drug, triplicate testing with careful quality control, and 
Point: Chemosensitivity Assays in Recurrent Ovarian Cancer

computer-assisted image analysis and result interpretation. The different assays are distinguished by their criteria for measuring drug effect. The ATP luminescence assay measures the effect of treatment on the ATP content of cancer cells using the luciferinluciferase assay. The MiCK assay uses an automated microculture kinetic assay of drug-induced apoptosis to evaluate chemosensitivity. The ChemoFx assay uses computer image analysis to count and calculate the surviving cell fraction and create a dose-response curve.

\section{Evidence of the Predictive Value of In Vitro Chemosensitivity Testing}

The microplate (ATP) luminescence assay was shown to be predictive in several correlative retrospective studies and a recent prospective trial reported by Cree et $\mathrm{al}^{8}{ }^{8}$ The latter randomized trial of 180 woman with recurrent platinum-resistant ovarian cancer compared the response rate and progression-free survival in patients treated according to an ATP-based tumor chemosensitivity assay and those treated according to physician's choice. In this trial, 86 women were randomized to physician's-choice chemotherapy and 94 to assay-directed therapy. The response was assessable in 147 patients, with $31.5 \%$ experiencing a partial or complete response compared with $40.5 \%$, respectively. Increased use of combination therapy was seen in the physician's-choice arm during the study, presumably as a result of the observed effects of assay-directed therapy. Patients entering the physician's-choice arm of the study during the first year had significantly worse outcomes than those who entered in the subsequent years, supporting the concept that physician drug selection was influenced by a learning curve from the assay results (hazard ratio, 0.44; 95\% CI, 0.2-0.9; $P<.03$ ). Although this was a small randomized clinical trial, it clearly documented a trend toward improved response and progression-free survival for assay-directed treatment. Although the ATP-based chemosensitivity assay showed potential benefit in this setting, it has limited availability in the United States.

The MiCK assay (DiaTech, Houston, Texas) measures drug-induced apoptosis using a proprietary technology. This assay uses a sophisticated algorithm to monitor and compute the amounts of apoptosis caused by each drug to establish a drug-sensitivity profile of the patient's tumor cells. In a recent ASCO presentation, a multicenter prospective trial of 128 women with advanced ovarian cancer tested the $\mathrm{MiCK}$ assay to evaluate its prediction of overall survival and response. ${ }^{9}$ The assay showed superior survival and response when the patient received a regimen with a MiCK apoptosis score greater than 1.7. Specifically, results showed a hazard ratio for death of 7.2 for apoptosis scores less than 1.7. Although these data are encouraging, they focus primarily on up-front treatment and do not completely address concerns about the use of chemosensitivity assays for recurrent cancer.

The most compelling evidence supporting the use of assay-directed therapy is from studies of the ChemoFx assay (Precision Therapeutics, Pittsburgh, Pennsylvania). Gallion et al. ${ }^{10}$ evaluated the value of this chemosensitivity assay test for predicting progression-free interval in 135 women with recurrent ovarian cancer. This multi-institutional retrospective study conducted between 1997 and 2002 divided the chemosensitivity into 3 discrete categories: responsive, intermediate, and nonresponsive. The results were highly significant $(P=.01)$, showing a 3 -fold increase in progression-free interval for patients treated with a drug that tested as responsive compared with those treated with a nonresponsive drug (Figure 1).

Although these progression-free interval data ${ }^{10}$ are important evidence that assay-selected therapy is predictive of potential clinical benefit, a study of overall survival is also an important measure of value. In an editorial Cannistra ${ }^{11}$ makes the point that there is a lack of evidence supporting the use of progression-free survival as a surrogate for overall survival in the recurrent ovarian cancer setting, and argues that overall survival remains the only valid end point in studies of recurrent ovarian cancer. To evaluate the value of chemosensitivity testing in predicting overall survival, Herzog et al. ${ }^{12}$ retrospectively studied 192 women at participating institutions with ovarian cancer who underwent chemosensitivity assay testing using ChemoFx. The primary objective was to determine the association between overall survival for patients with stages III/ IV primary ovarian cancer treated with platinumbased therapy and the responsiveness of their tumor specimens to platinum agents, as determined using ChemoFx. The median overall survival was 72 months for patients who underwent treatment classified as responsive, 49 months for patients who had 
Schink and Copeland

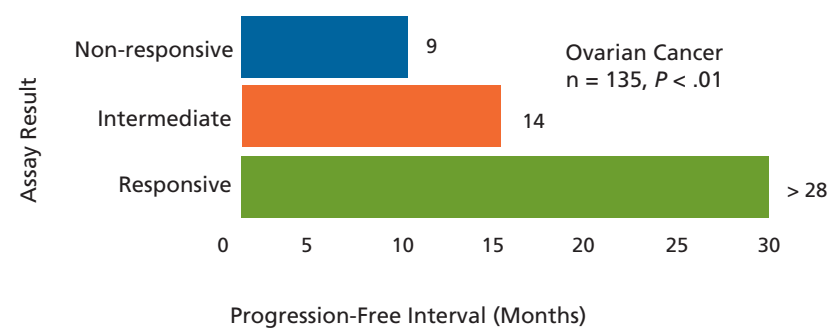

Figure 1 Progression-free survival based on chemosensitivity assay result.

Data from Gallion H, Christopherson WA, Coleman RL, et al. Progression-free interval in ovarian cancer and predictive value of an ex vivo chemoresponse assay. Int J Gynecol Cancer 2006;16:194-201.

an intermediately responsive result, and 28 months for those with a nonresponsive result $(P=.0386$, Wilcoxon test; Figure 2).

\section{In Vivo Versus In Vitro Testing}

These retrospective studies clearly document that chemosensitivity assays in general, and the Che$\mathrm{moFx}$ assay in particular, predict progression-free survival in recurrent ovarian cancer and overall survival in ovarian cancer. Although these data may not convince every clinician to rely on assays when selecting treatment, all clinicians use a form of chemosensitivity testing. The question is, do clinicians rely on laboratory in vitro testing or in vivo real-life testing? When relying solely on the patient's in vivo response to treatment as measured through examination, CA-125 level, and imaging, most patients are treated with a drug that is more likely to fail than to succeed. The drugs NCCN recommends for treating recurrent disease typically have response rates of $6 \%$ to $25 \%$. Researchers at Northwestern recently studied the question of whether response to one salvage drug predicts the response to another in platinumresistant ovarian cancer, and found that it was not predictive. ${ }^{13}$ This was a retrospective analysis of 114 women with primary or secondary platinum-resistant ovarian cancer. All women were treated with both monthly liposomal doxorubicin and weekly topotecan in sequence as first salvage single-agent chemotherapy after developing platinum-resistant recurrent ovarian cancer. In this cohort of women, the response rate to liposomal doxorubicin was $25 \%$, and $24 \%$ to weekly topotecan. A response to either treatment, but not to both, was seen in $44 \%$ of patients. Only $4 \%$ of patients experienced response to

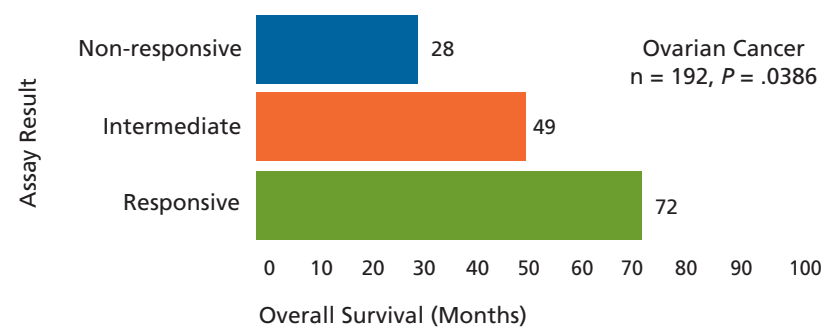

Figure 2 Overall survival based on assay results. Data from Herzog TJ, Krivak TC, Fader AN, Coleman RL. Chemosensitivity testing with ChemoFx and overall survival in primary ovarian cancer. Am J Obstet Gynecol 2010;203: 68.e1-6.

both drugs, in contrast to the $44 \%$ who experienced response to only one (Figure 3). Although nearly half of the patients experienced benefit from chemotherapy, no clinical factors in the platinum-resistant patient cohort predicted which drug would yield a response. Ideally, laboratory data would guide this drug choice so the patient is first offered the drug most likely to provide clinical benefit. ChemoFx, the most commonly used chemosensitivity in vitro assay, has a 0.70 correlation with clinical response, which is clearly better than the $25 \%$ empiric selection. ${ }^{14}$

\section{Can We Continue to Rely Solely on Clinical Features in Our Selection of Salvage Therapy?}

Prognostic factors for women with recurrent ovarian cancer include histology, CA-125 level, radiographic versus clinical disease, and treatment-free interval. ${ }^{15}$ In 1989, Blackledge et al. ${ }^{16}$ published a multivariate analysis of factors predicting response in 5 different phase II ovarian cancer clinical trials. He showed that the longer the interval from last treatment, the higher the probability of response, and that time to progression was more predictive of response than the treatment drug. In 1991, a retrospective analysis of women who experienced relapse after initial platinum-based chemotherapy was published, ${ }^{17}$ which evolved the concept of treatment-free interval by noting the predictive value of the "cisplatinum-free interval."

Those who disagree on this issue will likely argue that these prognostic factors are the paint on the oncologist's palette that create the art of clinical oncology. Surely, with the aid of laboratory information, patients can hope for more than creative inspiration. Similarly, criticisms of the retrospective nature of 
Point: Chemosensitivity Assays in Recurrent Ovarian Cancer

most of the data supporting the benefit of chemosensitivity assays ignore the fact that the concept of platinum-sensitivity was based on and adopted entirely from retrospective analyses.

In the past 2 decades the number of drugs with documented activity against ovarian cancer has increased dramatically, but the selection criteria has remained unchanged. The concept of platinum resistance is a gross simplification of a complex biologic system and cannot remain the primary selection criterion for choosing salvage chemotherapy. One significant flaw in this definition is the lack of consensus regarding evidence for disease recurrence: whether it is a specific CA-125 level, a percentage CA-125 increase, a radiographic finding, or the onset of symptoms. Should clinicians believe that a woman with multifocal recurrent disease 8 months after an extensive surgical effort leaving no visible residual disease is more likely to experience response to a subsequent platinum-based chemotherapy regimen than a woman who undergoes a suboptimal "peek and shriek" surgery who manifests an isolated recurrence at 5 months? After all, when did this recurrence actually occur? A recent EORTC study ${ }^{18}$ shows that an average of 5 months separates the significant rise in CA-125 (twice the normal limit) from the onset of clinical symptoms, and that early intervention with empirically selected chemotherapy did not impact overall survival. The question remains whether this lack of a survival benefit with early treatment was a reflection of tumor biology, an endorsement for prolonging the platinum-free interval, or confirmation that when using empiric drug selection the response rate is so low that benefit is minimal.

\section{How Much Retrospective Data Provide Enough Evidence to Accept the Value of a Laboratory Test?}

The goal of personalized medicine is accurate prediction of a patient's response to treatment. In an ideal world this evidence would be generated by a prospective trial. In the case of ovarian cancer, a cooperative group such as the Gynecologic Oncology Group (GOG) should perform a prospective randomized trial involving chemosensitivity assaydirected salvage therapy. Although the cooperative groups have discussed opening a trial such as this, no study has been initiated. Precision Therapeutics

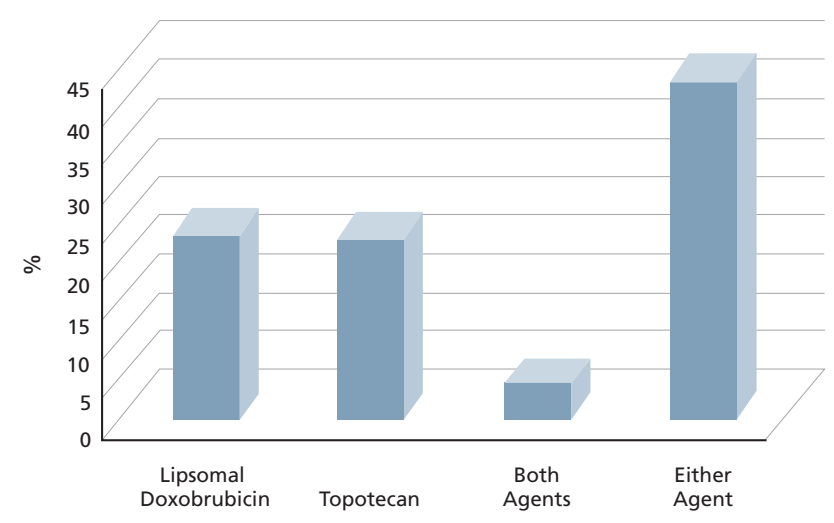

Figure 3 Response to salvage chemotherapies in platinumresistant ovarian cancer at Northwestern University.

From Morgan J, Berry E, Lurain JR, et al. Sequential liposomal doxorubicin and topotecan salvage chemotherapy in platinumresistant epithelial ovarian cancer: response to one drug dose does not predict response to the other. Presented at Western Association of Gynecologic Oncologists 38th Annual Meeting; June 24-27, 2009; Vancouver, British Columbia. Courtesy of Jacqueline Morgan, MD, University of Kansas School of Midicine, Wichita, KS.

is currently conducting a prospective noninterventional study of the association between in vitro drug response and clinical outcomes in women with recurrent epithelial ovarian cancer. This study, PT 301, allows clinicians to choose the salvage therapy and compares the outcomes with the assay results. The dearth of prospective trial data leaves a glaring hole in the usual evidence paradigm but must not be confused with evidence of no benefit. Without this level 1 evidence, the bulk of compelling laboratory and retrospective evidence that has shown patient benefit cannot be ignored. In colon cancer, the value of Kras testing to predict the benefit of using cetuximab was readily accepted based on a single retrospective study. Trastuzumab was adopted for the treatment of HER2-positive breast cancer based initially on retrospective data. With more than a dozen NCCN-acceptable treatments for recurrent ovarian cancer, and only the platinum-free interval to guide decision-making, the results of these studies support including chemosensitivity assay results in salvage therapy decision-making whenever feasible.

\section{Conclusions}

Using highly technical scientific and engineering principles, chemosensitivity assay testing has evolved in the past decade to produce clinically reliable re- 
sults that predict progression-free and overall survival. The opportunity to personalize therapy in the setting of recurrent platinum-resistant ovarian cancer through using assay-directed therapy should not be overlooked. Patients deserve the best prospect for treatment response, and chemosensitivity assay testing offers the chance to avoid ineffective therapies.

\section{References}

1. Jemal A, Siegel R, Xu J, Ward E. Cancer statistics, 2010. CA Cancer J Clin 2010;60:277-300.

2. Schilsky RL. Personalizing cancer care: American Society of Clinical Oncology presidential address 2009. J Clin Oncol 2009;27:3725-3730.

3. Salmon SE, Hamburger AW, Soehnlen B, et al. Quantitation of differential sensitivity of human-tumor stem cells to anticancer drugs. N Engl J Med 1978;298:1321-1327.

4. Von Hoff DD. He's not going to talk about in-vitro predictive assays again, is he? J Natl Cancer Inst 1990;82:96-101.

5. Loizzi V, Chan JK, Osann K, et al. Survival outcomes in patients with recurrent ovarian cancer who were treated with chemoresistance assayguided chemotherapy. Am J Obstet Gynecol 2003;189:1301-1307.

6. Karam AK, Chiang JW, Fung E, et al. Extreme drug resistance assay results do not influence survival in women with epithelial ovarian cancer. Gynecol Oncol 2009;114:246-252.

7. Matsuo K, Eno ML, Im DD, et al. Clinical relevance of extent of extreme drug resistance inepithelial ovarian carcinoma. Gynecol Oncol 2010;116:61-65.

8. Cree IA, Kurbacher CM, Lamont A, et al. A prospective randomized controlled trial of tumour chemosensitivity assay directed chemotherapy versus physician's choice in patients with recurrent platinum-resistant ovarian cancer. Anticancer Drugs 2007;18:1093-1101.
9. Salom EM, Penalver M, Homesley HD, et al. Can we increase response rate (RR) and overall survival (OS) by individualizing chemotherapy in ovarian cancer $(\mathrm{OC})$ : the role of a new chemotherapy $(\mathrm{CT})$ induced apoptosis assay [abstract]. J Clin Oncol 2010;28(Suppl 1):Abstract 5112.

10. Gallion H, Christopherson WA, Coleman RL, et al. Progressionfree interval in ovarian cancer and predictive value of an ex vivo chemoresponse assay. Int J Gynecol Cancer 2006;16:194-201.

11. Cannistra SA. Evaluating new regimens in recurrent ovarian cancer: how much evidence is good enough? J Clin Oncol 2010;28:3101-3103.

12. Herzog TJ, Krivak TC, Fader AN, Coleman RL. Chemosensitivity testing with ChemoFx and overall survival in primary ovarian cancer. Am J Obstet Gynecol 2010;203:68.e1-6.

13. Morgan J, Berry E, Lurain JR, et al. Sequential liposomal doxorubicin and topotecan salvage chemotherapy in platinum-resistant epithelial ovarian cancer: response to one drug dose does not predict response to the other. Presented at Western Association of Gynecologic Oncologists 38th Annual Meeting; June 24-27, 2009; Vancouver, BC.

14. Ness RB, Wisniewski SR, Eng H, Christopherson W. Cell viability assay for drug testing in ovarian cancer: in vitro kill versus clinical response. Anticancer Res 2002;22(2B):1145-1149.

15. Markman M, Bookman MA. Second-line treatment of ovarian cancer. Oncologist 2000;5:26-35.

16. Blackledge G, Lawton F, Redman C, Kelly K. Response of patients in phase II studies of chemotherapy in ovarian cancer: implications for patient treatment and the design of phase II trials. Br J Cancer 1989;59:650-653.

17. Markman $M$, Rothman R, Hakes $T$, et al. Second-line platinum therapy in patients with ovarian cancer previously treated with cisplatin. J Clin Oncol 1991;9:389-393.

18. Rustin GJ, van der Burg ME. A randomized trial in ovarian cancer (OC) of early treatment of relapse based on CA125 level alone versus delayed treatment based on conventional clinical indicators (MRC OV05/EORTC 55955 trials) [abstract]. J Clin Oncol 2009;27(Suppl 1):Abstract 1 